\section{Dysphagie: Tumorangst meist unberechtigt}

\begin{abstract}
Bei einer Dysphagie ohne Alarmsymptome kann man finnischen Forschern zufolge davon ausgehen, dass kein malignes Geschehen dahintersteckt. Die Wissenschaftler haben einen Algorithmus entwickelt, der helfen soll, unnötige Diagnostik einzusparen.
\end{abstract}

Z ur Abklärung einer Dysphagie steht eine ganze Armada diagnostischer Maßnahmen zur Verfügung, von der einfachen endoskopischen Untersuchung (EG, Ösophagogastroskopie) über die Videofluorografie (VFG) und die Manometrie bis hin zur endoskopischen Untersuchung des Schluckvorgangs mittels FEES (flexible endoscopic evaluation of swallowing). Bei Weitem nicht alle davon sind im Einzelfall sinnvoll, wie eine Studie aus Finnland zeigt. Nach Pia Nevalainen und Kollegen von der Universitätsklinik in Helsinki kann man sich vor allem eine aufwendige Tumorsuche in den meisten Fällen sparen.

Die Forscher haben aus der Datenbank ihrer HNO-Klinik 303 Patienten herausgegriffen, die im Jahr 2009 wegen einer Dysphagie in ihre Klinik überwiesen worden waren. Um herauszufinden, ob die Schluckstörung ein frühes Symptom einer malignen Erkrankung darstellte, verlinkte man diese Fälle mit dem finnischen Krebsregister. Die Auswertung erstreckte sich dabei über drei Jahre. Wie Nevalainen und Kollegen berichten, konnten die Patienten im Nachhinein in fünf Diagnosegruppen eingeteilt werden: $-55 \%$ hatten eine rein deskriptive Diagnose einer Dysphagie erhalten (,unspezifische Dysphagie ohne andere Zeichen oder Symptome").

_-In $17 \%$ lag eine gastroösophageale Reflux-Erkrankung (GERD) vor.

_In $11 \%$ ließ sich eine neurologische Ursache für die Beschwerden ausmachen. - $9 \%$ hatten ein Zenker-Divertikel und _bei insgesamt $8 \%$ lagen ,andere Ursachen" zugrunde.

In der letzteren Gruppe fanden sich 18 Patienten, bei denen sich die Probleme auf bereits behandelte Kopf-Hals-Tumoren oder eine Operation in diesem Bereich zurückführen ließen. Ein (bislang unentdeckter) maligner Tumor lag in fünf Fällen $(0,02 \%)$ vor, wobei die Verdachtsdiagnose in allen Fällen auf der Hand lag: Bei dreien hatten sich die
Schluckbeschwerden fortschreitend entwickelt; die Patienten berichteten über steckengebliebene Essensreste in der Speiseröhre. Hier konnte die Diagnose mithilfe einer EG gestellt werden. Bei den restlichen beiden offenbarte sich der Befund - ein oropharyngealer bzw. ein laryngealer Tumor - bereits bei der klinischen Untersuchung.

In der HNO-Klinik war eine ganze Reihe diagnostischer Verfahren zum Einsatz gekommen, am häufigsten die Videofluorografie $(n=173)$; diese erbrachte jedoch in 117 Fällen normale Ergebnisse. Hals-Ultraschall $(\mathrm{n}=36)$ und FEES ( $n=55)$ wurden am zweithäufigsten eingesetzt; dabei war Ersterer in der Regel unauffällig. Ein anomaler Befund zeigte sich dagegen bei 30 der mittels FEES untersuchten Patienten, wobei neurologische Probleme, aber auch Kopf-Hals-Tumoren gefunden wurden.

Fazit: Auf der Grundlage ihrer Erkenntnisse schlagen die Autoren nun einen diagnostischen Algorithmus vor: Alarmsignale, die auf eine mögliche Tumorerkrankung hinweisen, sind demnach: steckengebliebenes Essen, Gewichtsverlust und fortschreitende Symptomatik. Diese erfordern, so Nevalainen et al., eine umgehende Evaluation. Zur Hochrisikogruppe gehören insbesondere Patienten über 50, die exzessiv rauchen oder trinken.

Dagegen ist ein Tumor höchst unwahrscheinlich, wenn sich bei der klinischen Untersuchung keine Auffälligkeiten finden und die genannten Alarmsignale fehlen. Wie die Drei-Jahres-Daten zeigten, mündete kein Fall einer „unspezifischen Dysphagie ohne weitere Zeichen und Symptome" in einer Tumorerkrankung.

Die sorgfältige Anamnese gekoppelt mit der klinischen Untersuchung ist den Forschern zufolge die Basis der Dysphagie-Diagnostik. Allein durch Befragen des Patienten könne man herausfinden, ob es sich um eine orale, pharyngeale oder ösophageale Dysphagie handle.

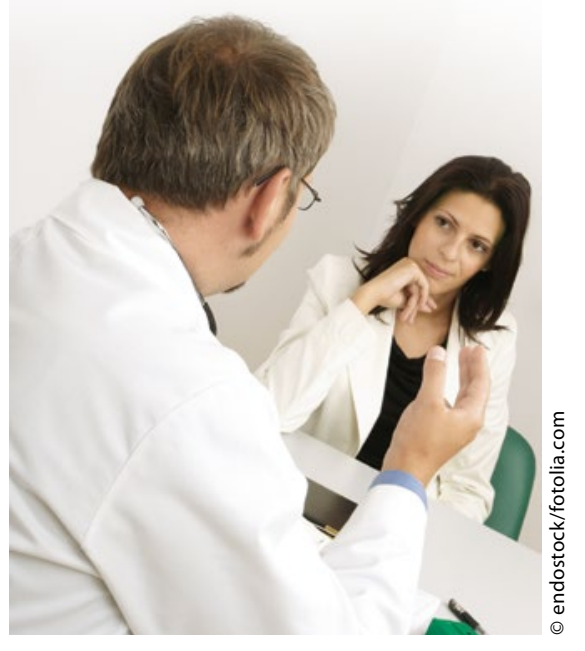

Bereits durch eine sorgfältige Anamnese lässt sich das Spektrum möglicher Dysphagie-Ursachen deutlich eingrenzen.

Das Fehlen einer offenkundigen Ursache bei oraler Dysphagie mache eine neurologische Störung wahrscheinlich. Patienten mit Dysphagie im Bereich des Rachens zeigten Aspiration, Regurgitation, einen insuffizienten velopharyngealen Verschluss oder hätten Schwierigkeiten, den Bolus in den Ösophagus zu befördern. Und die ösophageale Dysphagie sei durch Sodbrennen gekennzeichnet oder dadurch, dass das Essen retrosternal steckenbleibe. Ein gastroösophagealer Reflux war die häufigste Ursache für die Beschwerden, wenn die Patienten über Sodbrennen berichteten und auf Protonenpumpenhemmer ansprachen.

Endoskopie, Ösophagusmanometrie und VFG gelten gemeinhin als wertvollste Diagnose-Tools, wobei die mit der VFG verbundene Strahlenlast berücksichtigt und die Indikation entsprechend sorgfältig gestellt werden müsse. In der Studie zeigte die VFG den größten Nutzen in der Diagnostik von Zenker-Divertikel und Dysfunktion des M. cricopharyngeus Störungen, an denen allerdings nur wenige Patienten litten. Der Ultraschall wurde häufig offenbar nur eingesetzt, um die Patienten zu beruhigen; ohne tastbare Umfangsvermehrung ist der Nutzen laut Nevalainen und Kollegen fraglich.

Die Autoren betonen, dass nach drei Jahren bei jedem zweiten Patienten die Symptome verschwunden waren oder sich deutlich abgeschwächt hätten; dies zeige das „Potenzial für einen spontan günstigen Verlauf“. Dr. Elke Oberhofer

Nevalainen P et al. Dysphagia and Malignancy: A Three-Year Follow-Up and Survey of National Cancer Registry Data. Laryngoscope 2015, online 21. Dezember 2015 